\title{
Benefícios da posição de prona em pacientes com COVID-19 não-intubados
}

\author{
Benefits of the prone position in patients with non-intubated COVID-19 \\ Beneficios de la posición prona en pacientes con COVID-19 no intubado
}

Recebido: 09/05/2021 | Revisado: 13/05/2021 | Aceito: 16/05/2021 | Publicado: 04/06/2021

Larissa Toloy Bigaran

ORCID: https://orcid.org/0000-0001-5815-4000

Universidade Brasil, Brasil

E-mail: larissatoloyb@gmail.com

Layssa Delgado Meira

ORCID: https://orcid.org/0000-0001-7956-931X União das Faculdades dos Grandes Lagos, Brasil E-mail: layssameira7@gmail.com

Juliana Valões Alencar da Silva

ORCID: https://orcid.org/0000-0001-6006-5322 União das Faculdades dos Grandes Lagos, Brasil E-mail: juliana.valencar@gmail.com

Vítor Graça Rêgo

ORCID: https://orcid.org/0000-0001-8514-1838 União das Faculdades dos Grandes Lagos, Brasil E-mail: vitor.graca@hotmail.com

Talita Costa Barbosa

ORCID: https://orcid.org/0000-0002-0396-0651 Universidade Brasil, Brasil

E-mail: talitacostabarbosa@gmail.com

Elenberg Chaves de Paula

ORCID: https://orcid.org/0000-0003-4170-7726

União das Faculdades dos Grandes Lagos, Brasil

E-mail: elenbergde@hotmail.com

\section{Resumo}

O objetivo do estudo foi evidenciar os benefícios da posição de prona em pacientes com COVID-19 não-intubados. Trata-se de uma revisão integrativa da literatura, usando os descritores "prone position" and "coronavirus" not "respiration, artificial", usando as bases de dados PubMed, o Google Scholar, a Biblioteca Virtual em Saúde (BVS), Literatura Latino-Americana e do Caribe em Ciências da Saúde (LILACS). Após aplicação dos critérios de inclusão e exclusão, utilizou-se o fluxograma PRISMA para elucidar a seleção dos artigos e uma tabela mostrando os resultados que compõem a revisão. A posição de prona em pacientes acometidos pelo SARS-COV-2 em ventilação não-invasiva trouxe principalmente uma redução do esforço respiratório e com isso foi relatado que a mesma levou a uma menor incidência de intubação. Ainda, a mesma pode melhorar a homogeneidade pulmonar, a redistribuição pulmonar, a complascência pulmonar, a proporção de recrutamento para infiltração e o recrutamento de pulmão. Conclui-se, que a posição de prona proporciona diversos benefícios para os pacientes não-intubados com COVID-19, proporcionando melhora da saturação e redução da mortalidade, além de prevenir a intubação.

Palavras-chave: Decúbito ventral; Infecções por coronavírus; Ventilação não invasiva, COVID-19.

\begin{abstract}
The aim of the study was to highlight the benefits of the prone position in patients with unintubated COVID-19. This is an integrative literature review, using the keywords "prone position" and "coronavirus" not "respiration, artificial", using the PubMed databases, Google Scholar, the Virtual Health Library (VHL), Latin Literature -American and Caribbean Health Sciences (LILACS). After applying the inclusion and exclusion criteria, the PRISMA flowchart was used to elucidate the selection of articles and a table showing the results that make up the review. The prone position in patients affected by SARS-COV-2 on non-invasive ventilation has mainly brought about a reduction in respiratory effort and it has been reported that it has led to a lower incidence of intubation. Furthermore, it can improve pulmonary homogeneity, pulmonary redistribution, lung compliance, the proportion of recruitment for infiltration and lung recruitment. In conclusion, the prone position provides several benefits for patients not intubated with COVID19, providing improved saturation and reduced mortality, in addition to preventing intubation.
\end{abstract}

Keywords: Prone position; Coronavírus infections; Noninvasive ventilation; COVID-19. 


\begin{abstract}
Resumen
El propósito del estudio fue resaltar los beneficios de la posición prona en pacientes con COVID-19 no intubado. Esta es una revisión de literatura integradora, utilizando las palabras clave "posición prona" y "coronavirus" no "respiración, artificial", utilizando las bases de datos PubMed, Google Scholar, la Biblioteca Virtual en Salud (BVS), Literatura Latina-Ciencias de la Salud de América y el Caribe ( LILACS). Luego de aplicar los criterios de inclusión y exclusión, se utilizó el diagrama de flujo PRISMA para dilucidar la selección de artículos y una tabla que muestra los resultados que componen la revisión. La posición de decúbito prono en pacientes afectados por SARS-COV-2 en ventilación no invasiva trajo principalmente una reducción en el esfuerzo respiratorio y se informó que condujo a una menor incidencia de intubación. Además, puede mejorar la homogeneidad pulmonar, la redistribución pulmonar, la distensibilidad pulmonar, la proporción de reclutamiento para la infiltración y el reclutamiento pulmonar. En conclusión, la posición prona proporciona varios beneficios para los pacientes no intubados con COVID-19, proporcionando una mejor saturación y una reducción de la mortalidad, además de prevenir la intubación.
\end{abstract}

Palabras clave: Posición prona; Infecciones por coronavírus; Ventilación no invasiva; COVID-19.

\title{
1. Introdução
}

Os pacientes com doença coronavírus (COVID-19) estão em risco de síndrome do desconforto respiratório agudo. Tais pacientes intubados acometidos por essa síndrome, o posicionamento prono precoce e prolongado tem melhorado a saturação e reduzido a mortalidade. Diante disso, a posição prona tem se mostrado útil para associar ao tratamento desses pacientes (Elharrar et al., 2020).

O COVID-19 tem aumentado o número de pacientes internados no hospital com quadro de insuficiência respiratória. Grande parte desses pacientes necessitam de um suporte ventilatório não invasivo. Vale ressaltar que a taxa de piora do quadro é muito alta e a intubação faz-se necessária, saturando rapidamente os recursos de saúde e a disponibilidade de leitos em unidades de terapia intensiva (UTI). Dessa maneira, tem-se um aumento na mortalidade. A síndrome do desconforto respiratório (SRDA) é uma das principais complicações da COVID-19. Seu tratamento requer intubação traqueal e ventilação mecânica, e os pacientes podem se beneficiar com o posicionamento prono. Este tem demonstrado obter uma melhor oxigenação e reduzir a mortalidade na síndrome não relacionada ao COVID-19 (Coppo, et al., 2020).

Tal situação está relacionada com o aumento da oxigenação e é devido a melhor combinação ventilação-perfusão na posição prona, porque as áreas dorsais não são mais comprimidas pelo peso da cavidade abdominal e do mediastino, e podem reabrir, levando ao recrutamento de regiões mais eficientes na troca gasosa. O benefício da mortalidade não pode ser explicado apenas pela melhora da oxigenação e tem sido associado à diminuição da superdistenção e do recrutamento alveolar cíclico nas respirações correntes, reduzindo o risco de lesão pulmonar induzida pelo ventilador (Coppo, et al., 2020).

A ventilação prona refere-se ao fornecimento da ventilação mecânica ao paciente deitado em posição prona, o qual é usado para SRDA como estratégia para melhoria da saturação e foi proposto pela primeira vez na década de 1970 como método de auxílio para trocas gasosas. Tal fato, deve-se para reduzir a atelectasia em pulmões lesados, diminuindo os gradientes de pressão pleural e restaurando a aeração para os segmentos dorsais do pulmão. A posição prona foi estabelecida como estratégia de resgate para hipoxemia grave. Os pacientes graves devem ter o procedimento iniciado precocemente, idealmente dentro de 36 a 48 horas e mantido por 18 a 20 horas consecutivas. Os melhores resultados são quando usados em combinação com baixo volume corrente e bloqueio neuromuscular (Petrone et al., 2020)

Diante disso, destaca-se a importância da seguinte pesquisa pelo fato de abordar as informações sobre uma alternativa complementar ao tratamento desses pacientes com SRDA acometidos pelo coronavírus. Dessa maneira, tal estudo pode proporcionar a elaboração de futuros estudos voltados para o assunto ou pesquisas relacionadas as tema abordado, para uma melhoria na qualidade da assistência para os pacientes infectados pela COVID-19. Além disso, é um grande problema vivenciado pela saúde mundial, diante do estado pandêmico que todos estão vivendo. Sendo assim, surgiu a seguinte questão norteadora: Qual a importância e os benefícios da posição de prona em pacientes com COVID-19 não-intubados?

Em virtude disso, objetivou-se analisar quais são os benefícios da posição prona em pacientes acometidos pela 
COVID-19 e que não foram intubados.

\section{Metodologia}

O estudo realizado foi uma revisão integrativa da literatura, descrita como um método que proporciona realizar a síntese de conhecimento e aplicar os resultados de estudos significativos na prática (Souza et al., 2010). Os descritores utilizados foram "prone position" and "coronavirus" not "respiration, artificial". Foram encontrados cem artigos e desses selecionados vinte, nas línguas inglesa, espanhola e portuguesa. Para alcance dos estudos escolhidos, as plataformas usadas foram a PubMed, o Google Scholar e a Biblioteca Virtual em Saúde (BVS), a qual possui diversas bases de dados, entre elas, foram selecionadas a Literatura Latino-Americana e do Caribe em Ciências da Saúde (LILACS).

Em relação aos critérios de inclusão e exclusão, utilizou-se como de critérios de inclusão: artigos disponíveis com publicação nos idiomas português, espanhol e/ou inglês, que abordassem o tema do estudo e que tivessem sido publicados nos anos a qual se decretou a pandemia do novo coronavírus (final de 2019-2021). A exclusão baseou-se nos artigos nos quais citavam a posição de prona usada apenas em pacientes intubados, ou também aquelas que não se apresentavam com diagnóstico de COVID-19.

\section{Resultados e Discussão}

Através das buscas efetivadas nas bases de dados, encontrou-se 21 artigos na PubMed, 34 artigos na Lilacs, 12 artigos na BVS e 158 artigos na Google Scholar, totalizando 225 artigos, que após aplicação dos critérios de inclusão e exclusão já relatados, restaram 180 documentos, dos quais, 20 estudos foram utilizados para a confecção da seguinte revisão, sendo esses, em língua inglesa e espanhola, 7 artigos retirados na base PubMed, 5 na base BVS, 3 na base LILACS e 5 artigos retirado na base Google Scholar.

Para melhor esclarecimento de todo método de busca e escolha dos artigos utilizados, optou-se pelo uso de um fluxograma baseado no protocolo PRISMA, ilustrado na Figura 1. 
Figura1 - Fluxograma demostrando a escolha dos artigos.

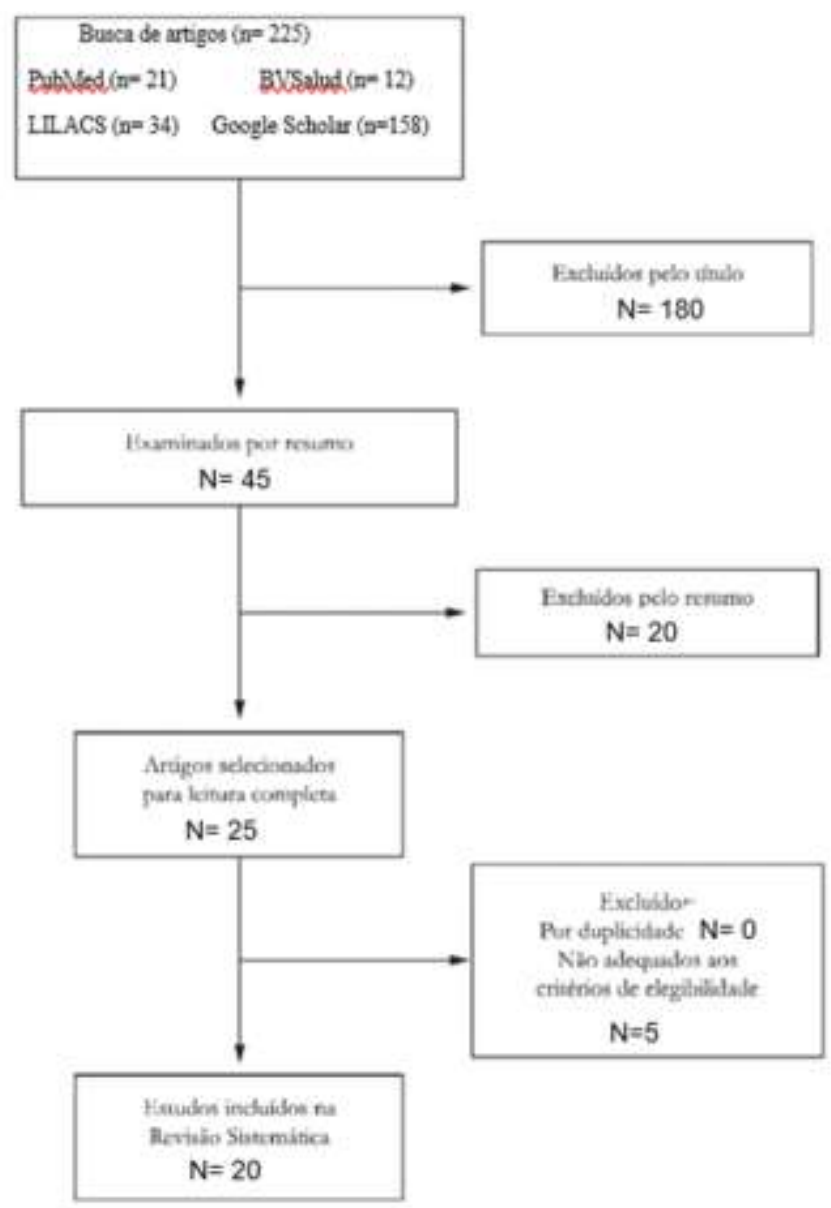

Fonte: Protocolo PRISMA adaptado.

Consecutivamente, realizou-se a leitura dos artigos escolhidos, inserindo em uma tabela (Quadro 1), os seguintes elementos: autor, ano, título, objetivo e perfis das amostras, usados para a organizar e demonstrar as informações colhidas para elaboração desta revisão.

Quadro 1 - Artigos utilizados para a revisão.

\begin{tabular}{|c|c|c|c|}
\hline $\begin{array}{ll}\text { AUTORES } & \text { E } \\
\text { ANO } & \end{array}$ & TITULO & OBJETIVO & PERFIL DA AMOSTRA \\
\hline $\begin{array}{l}\text { Coppo, A. et al., } \\
2020\end{array}$ & $\begin{array}{l}\text { Feasibility and physiological effects } \\
\text { of prone positioning in non- } \\
\text { intubated patients with acute } \\
\text { respiratory failure due to COVID-19 } \\
\text { (PRON-COVID): a prospective } \\
\text { cohort study }\end{array}$ & $\begin{array}{llr}\text { Investigar a viabilidade e o efeito } \\
\text { na troca } & \text { gasosa quando os } \\
\text { pacientes } & \text { acordados } \text { e r não } \\
\text { intubados } & \text { com } & \text { pneumonia } \\
\text { relacionada } & \text { a COVID-19 } & \text { são } \\
\text { colocados em posição de prona. }\end{array}$ & $\begin{array}{l}\text { Realizado com pacientes com idade } \\
\text { entre } 18 \text { e } 75 \text { anos, internados no } \\
\text { Hospital San Gerardo, Monza, Itália. } \\
\text { Foram recrutados } 56 \text { pacientes, entre } \\
20 \text { de março e } 9 \text { de abril de } 2020 \text {, dos } \\
\text { quais o posicionamento prono era } \\
\text { viável em } 47 \text { deles. }\end{array}$ \\
\hline $\begin{array}{l}\text { Elharrar, X. et al., } \\
2020\end{array}$ & $\begin{array}{l}\text { Use of Prone Positioning in } \\
\text { Nonintubated Patients With } \\
\text { COVID-19 and Hypoxemic Acute } \\
\text { Respiratory Failure }\end{array}$ & $\begin{array}{l}\text { Avaliar a viabilidade, eficácia e } \\
\text { tolerância da posição de prona em } \\
\text { pacientes acordados com COVID- } \\
19 \text { fora da UTI. }\end{array}$ & $\begin{array}{l}\text { Realizado com pacientes acordados, } \\
\text { não intubados, respirando } \\
\text { espontaneamento com COVID-19 e } \\
\text { insuficiência respiratória hipoxêmica, } \\
\text { internados no Hospital Aix-en- } \\
\text { Provence (França) de } 27 \text { de março a } 8 \\
\text { de abril de 2020. Os pacientes } \\
\text { selecionados e considerados elegíveis } \\
\text { foram os que precisassem de } \\
\text { suplementação de oxigênio e os com } \\
\text { achados sugestivos de COVID-19 na } \\
\text { tomografia de tórax. }\end{array}$ \\
\hline
\end{tabular}




\begin{tabular}{|c|c|c|c|}
\hline $\begin{array}{llll}\text { Jiang, } & \text { L.G } & \text { et } & \text { al., } \\
2020 & & & \end{array}$ & $\begin{array}{l}\text { "Conscious proning: An } \\
\text { introduction of a proning protocol } \\
\text { for non-intubated, awake, hypoxic } \\
\text { Emergency Department COVID-19 } \\
\text { patients" }\end{array}$ & $\begin{array}{l}\text { Criar um protocolo, baseado nas } \\
\text { evidências existentes, para } \\
\text { implementação da pronação no } \\
\text { departamento de emergência para } \\
\text { pacientes não acordados e não } \\
\text { intubados. }\end{array}$ & $\begin{array}{l}\text { Os dados inciais foram coletados de } \\
\text { mais de } 600 \text { pacientes com COVID-19, } \\
\text { onde foi relatado que a posição de } \\
\text { prona em pacientes acordados teve } \\
\text { efeitos bastante importantes na } \\
\text { oxigenação e heterogeneidade } \\
\text { pulmonar. }\end{array}$ \\
\hline $\begin{array}{l}\text { Ibarra-Estrada, M.A } \\
\text { et al., } 2020\end{array}$ & $\begin{array}{l}\text { Prone positioning in non-intubated } \\
\text { patients with COVID-19 associated } \\
\text { acute respiratory failure, the PRO- } \\
\text { CARF trial: A structured summary } \\
\text { of a study protocol for a randomised } \\
\text { controlled trial. }\end{array}$ & $\begin{array}{l}\text { Avaliar o efeito do } \\
\text { posicionamento prona na taxa de } \\
\text { intubação em pacientes acordados } \\
\text { portadores de COVID-19 e } \\
\text { insuficiência respiratória aguda. }\end{array}$ & $\begin{array}{l}\text { Todos os pacientes internados por } \\
\text { insuficiencia respiratória aguda } \\
\text { associada a infecção por coronavírus e } \\
\text { que necessitavam de oxigenação } \\
\text { suplementar no Hospital Civil de } \\
\text { Guadalajara e Hospital General do } \\
\text { Ocidente no México. }\end{array}$ \\
\hline $\begin{array}{l}\text { Wendt, C. et al., } \\
2021\end{array}$ & \begin{tabular}{lrr}
\multicolumn{3}{l}{ Prone Positioning of Patients With } \\
Coronavirus Disease 2019 Who Are \\
Nonintubated $\quad$ in & Hypoxic \\
Respiratory Distress: & Single-Site \\
Retrospective & Health & Records \\
Review & &
\end{tabular} & $\begin{array}{l}\text { Medir as variações apresentadas } \\
\text { na oximetria de pulso associadas } \\
\text { ao posicionamento prona em } \\
\text { pacientes portadores de infecção } \\
\text { por coronavírus no ano de } 2019 \text {. }\end{array}$ & $\begin{array}{l}\text { Revisou prontuários de pacientes } \\
\text { COVID-19 positivos em } 2019 \text { no } \\
\text { setor de emegência no período de } 30 \\
\text { de março de } 2020 \text { a } 30 \text { de abril de } \\
2020 .\end{array}$ \\
\hline $\begin{array}{l}\text { Whittemore, P. et al., } \\
2020\end{array}$ & $\begin{array}{l}\text { Use of awake proning to avoid } \\
\text { invasive ventilation in a patient with } \\
\text { severe COVID-19 pneumonitis }\end{array}$ & $\begin{array}{l}\text { Tratar o paciente em decúbito } \\
\text { vental o máximo possível, mas de } \\
\text { preferência em um período de } 18 \\
\text { horas por dia. }\end{array}$ & $\begin{array}{l}1 \text { homem asiático de } 60 \text { anos com } \\
\text { swab positivo para COVID-19 e com } \\
\text { alterações em vidro fosco visto em } \\
\text { tomografia de crânio atendido no } \\
\begin{array}{l}\text { departamento de acidentes } \\
\text { emergência. }\end{array}\end{array}$ \\
\hline Paul, V. et al., 2020 & $\begin{array}{l}\text { Proning in Non-Intubated (PINI) in } \\
\text { Times of COVID-19: Case Series } \\
\text { and a Review }\end{array}$ & $\begin{array}{l}\text { Discutir o uso da pronação em } 2 \\
\text { pacientes não-intubados, revisando } \\
\text { a litetura quanto a fisiologia da } \\
\text { ventilação e discutir o uso de tal } \\
\text { posição à beira leito. }\end{array}$ & $\begin{array}{lrr}2 \text { pacientes } & \text { com insuficiência } \\
\text { respiratória } & \text { hipoxêmica } & \text { aguda, } \\
\text { apresentando } & \text { melhoras } \\
\text { signitificativas na oxigenação, após a } \\
\text { posição de prona. }\end{array}$ \\
\hline $\begin{array}{l}\text { Burton-Papp, H.C et } \\
\text { al., } 2020\end{array}$ & $\begin{array}{l}\text { Conscious prone positioning during } \\
\text { non-invasive ventilation in COVID- } \\
19 \text { patients: experience from a single } \\
\text { centre. }\end{array}$ & $\begin{array}{l}\text { Avaliar o efeito do posicionamento } \\
\text { inclinado em pacientes em } \\
\text { ventilação não-invasiva. }\end{array}$ & $\begin{array}{l}\text { Analisaram via prontuário eletrônico } \\
\text { dados clínicos de } 81 \text { pacientes. }\end{array}$ \\
\hline $\begin{array}{l}\text { Bastoni, D. et al., } \\
2020\end{array}$ & $\begin{array}{l}\text { Prone positioning in patients treated } \\
\text { with non-invasive ventilation for } \\
\text { COVID-19 pneumonia in an Italian } \\
\text { emergency department. }\end{array}$ & $\begin{array}{l}\text { Tentar pronar pacientes COVID-19 } \\
\text { positivos internados no pronto- } \\
\text { socorro por apresentarem } \\
\text { insuficiência respiratória aguda e } \\
\text { usando ventilação não-invasiva } \\
\text { com capacetes de StarMed, } \\
\text { avaliando os efeitos da oxigenação } \\
\text { e nos sinais na ultrassom pulmonar. }\end{array}$ & $\begin{array}{l}\text { Selecionaram } 10 \text { pacientes, sendo } 8 \\
\text { homens e } 2 \text { mulheres, com idade } \\
\text { média de } 73 \text { anos. }\end{array}$ \\
\hline Raoof, S. et al., 2019 & 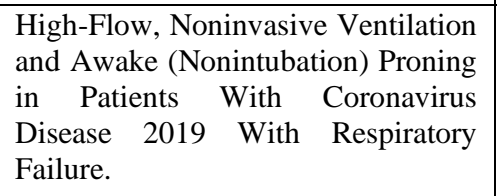 & $\begin{array}{l}\text { Descrever as indicações comuns, } \\
\text { contra-indicações e aspectos } \\
\text { práticos de aplicação da pronação } \\
\text { desperta, cânulas nasais de alto } \\
\text { fluxo e ventilação não invasiva. }\end{array}$ & $\begin{array}{l}20 \text { pacientes não intubados com } \\
\text { síndrome do desconforto respiratório } \\
\text { agudo moderada a grave, tratados } \\
\text { com } 2 \text { sessões de pronação por dia } \\
\text { por uma média de } 2 \text { horas cada. }\end{array}$ \\
\hline $\begin{array}{l}\text { Winck, } \\
\text { Ambrosino, N., } 2020\end{array}$ & $\begin{array}{l}\text { COVID-19 pandemic and non- } \\
\text { invasive respiratory management: } \\
\text { Every Goliath needs a David. An } \\
\text { evidence based evaluation of } \\
\text { problems. }\end{array}$ & $\begin{array}{l}\text { Decrever os problemas vinculados } \\
\text { ao tratamento da insuficiência } \\
\text { respiratória aguda induzida pela } \\
\text { infecção por coronavírus. }\end{array}$ & $\begin{array}{l}\text { Revisão de literatura usando as bases } \\
\text { de dados MEDLINE , EMBASE, } \\
\text { Cochrane Central Register of } \\
\text { Controlled Trials. }\end{array}$ \\
\hline $\begin{array}{l}\text { Sartini, C. et al., } \\
2020\end{array}$ & $\begin{array}{l}\text { Respiratory Parameters in Patients } \\
\text { With COVID-19 After Using } \\
\text { Noninvasive Ventilation in the } \\
\text { Prone Position Outside the Intensive } \\
\text { Care Unit. }\end{array}$ & $\begin{array}{l}\text { Relatar os parâmetros respiratórios } \\
\text { de após uma intervação em uma } \\
\text { séria de casos de pacientes com } \\
\text { COVID-19. }\end{array}$ & $\begin{array}{l}48 \text { pacientes tratados em UTIs e } 202 \\
\text { pacientes em enfermarias médicas, no } \\
\text { Instituto Científico San Raffaele, } \\
\text { Milão, Itália. }\end{array}$ \\
\hline
\end{tabular}




\begin{tabular}{|c|c|c|c|}
\hline $\begin{array}{l}\text { Araújo, M.S et al., } \\
2021\end{array}$ & $\begin{array}{l}\text { Prone positioning as an emerging } \\
\text { tool in the care provided to patients } \\
\text { infected with COVID-19: a scoping } \\
\text { review. }\end{array}$ & $\begin{array}{l}\text { Decrever as evidências científicas } \\
\text { disponíveis quanto a utilização da } \\
\text { posição de prona no tratamento ao } \\
\text { paciente com insuficiência } \\
\text { respiratória aguda provocada pela } \\
\text { COVID-19. }\end{array}$ & $\begin{array}{l}\text { Revisão de literatura usando sete } \\
\text { bases de dados, resultando em } 2441 \\
\text { estudos, dos quais } 12 \text { fizeram parte da } \\
\text { amostra. }\end{array}$ \\
\hline $\begin{array}{l}\text { Moghadam, V. D., } \\
\text { et al., } 2020\end{array}$ & $\begin{array}{l}\text { Prone positioning in management of } \\
\text { COVID- } 19 \text { hospitalized patients. }\end{array}$ & $\begin{array}{l}\text { Relatar os benefícios da ação do } \\
\text { decúbito ventral em pacientes com } \\
\text { Síndrome de r Desconforto } \\
\text { Respiratório Agudo por COVID- } \\
19 .\end{array}$ & $\begin{array}{l}10 \text { pacientes } \text { COVID-19 poitivos, } \\
\text { selecionados } \\
\text { especializados aleatoriamente, } \\
\text { específica para COVID-19, sendo } \\
70 \% \text { homens e } 30 \% \text { mulheres. }\end{array}$ \\
\hline $\begin{array}{l}\text { Instituto de } \\
\text { Efectividad Clínica y } \\
\text { Sanitaria, } 2020\end{array}$ & $\begin{array}{l}\text { Posicionamento en prono en } \\
\text { infección por COVID-19. }\end{array}$ & $\begin{array}{l}\text { Avaliar as evidências relacionadas a } \\
\text { eficácia e segurança da ventilação } \\
\text { em pacientes em posição de prona } \\
\text { que estão em tratamento por } \\
\text { COVID-19. }\end{array}$ & $\begin{array}{l}\text { Revisão de literatura usando bases de } \\
\text { dados, buscador genérico de internet } \\
\text { e financiadores de saúde. }\end{array}$ \\
\hline $\begin{array}{l}\text { Cohen, D. et al., } \\
2020\end{array}$ & $\begin{array}{l}\text { Beneficial effect of awake prone } \\
\text { position in hypoxaemic patients with } \\
\text { COVID-19: case reports and } \\
\text { literature review }\end{array}$ & $\begin{array}{l}\text { Revisar o conceito de posição de } \\
\text { prona acordada após apresentar } \\
\text { dois relatos de caso de pacientes } \\
\text { com COVID-19 moderada. }\end{array}$ & $\begin{array}{l}2 \text { pacientes COVID - } 19 \text { moderados } \\
\text { (diagnosticados por reação em cadeia } \\
\text { da polimerase nasal e da garganta) }\end{array}$ \\
\hline $\begin{array}{l}\text { Ghelichkhani P., } \\
\text { Esmaeili M., } 2020\end{array}$ & $\begin{array}{l}\text { Prone Position in Management of } \\
\text { COVID-19 Patients; a Commentary }\end{array}$ & $\begin{array}{l}\text { Revisar a literatura sobre o papel } \\
\text { da posição de prona no manejo de } \\
\text { paciente com COVID-19. }\end{array}$ & $\begin{array}{l}\text { Revisão de literatura nas principais } \\
\text { bases de busca. }\end{array}$ \\
\hline $\begin{array}{l}\text { Damarla, M. et al., } \\
2020\end{array}$ & $\begin{array}{l}\text { Prone Positioning of Nonintubated } \\
\text { Patients with COVID-19 }\end{array}$ & $\begin{array}{l}\text { Conduzir uma revisão retrospectiva } \\
\text { através de experiência em uma } \\
\text { série clínica de pacientes não } \\
\text { intubados. }\end{array}$ & $\begin{array}{l}9 \text { pacientes adultos em um centro } \\
\text { médico acadêmico } \\
\text { positivos }\end{array}$ \\
\hline $\begin{array}{l}\text { Dong, W. et al., } \\
2020\end{array}$ & $\begin{array}{l}\text { Early Awake Prone and Lateral } \\
\text { Position in Non-intubated Severe } \\
\text { and Critical Patients with COVID- } \\
19 \text { in Wuhan: A Respective Cohort } \\
\text { Study. }\end{array}$ & $\begin{array}{l}\text { Compartilhar as características } \\
\text { clínicas e os resultados de } \\
\text { pacientes graves e críticos. }\end{array}$ & $\begin{array}{l}25 \text { pacientes foram incluídos no } \\
\text { estudo. }\end{array}$ \\
\hline $\begin{array}{l}\text { Chad, T., Sampson, C., } \\
2020\end{array}$ & $\begin{array}{l}\text { Prone positioning in conscious } \\
\text { patients on medical wards: A review } \\
\text { of the evidence and its relevance to } \\
\text { patients with COVID-19 infection }\end{array}$ & $\begin{array}{l}\text { Ajudar os médicos da linha de } \\
\text { frente a decidir se adotar a posição } \\
\text { de prona pode ser benéfica para os } \\
\text { pacientes que estão tratando no } \\
\text { contexto da COVID-19. }\end{array}$ & $\begin{array}{l}\text { Revisão de literatura nas principais } \\
\text { bases de busca. }\end{array}$ \\
\hline
\end{tabular}

Fonte: Autores.

Segundo o estudo de Chad e Sampson (2020), em relação a posição prona, o paciente é reposicionado para ficar na posição deitada para frente, e o uso dessa técnica visa melhorar a oxigenação dos pacientes com insuficiência respiratória aguda inclui o paciente acordado, sem estar intubado, em que o momento da intubação foi adiado. A técnica é amplamente utilizada para uma terapia de resgate, principalmente em pacientes mecanicamente com síndrome do desconforto respiratório agudo grave. Dessa maneira, o ensaio clínico randomizado Posicionamento em Prona na Síndrome de Dificuldades Respiratórias Agudas Graves, relatada no estudo, apontou um benefício de mortalidade considerável em pacientes intubados com SDRA grave, quando essa técnica foi usada junto com ventilação pulmonar. Dessa maneira, encontra-se benefícios na mortalidade em pacientes adultos ventilados com SDRA. O tratamento prona por mais de 16 horas por dia resultou uma diferença na mortalidade versus não prona. Diante dos benefícios encontrados da posição prona, alguns estudos sugeriram que o benefício poderia se estender aos pacientes acometidos pela COVID-19 precoce nas enfermarias médicas.

Dong et al. (2020), relata em seu estudo que umas das características da SDRA nos pacientes graves seria a falta de homogeneidade pulmonar. Qualquer atraso no tratamento pode causar uma menor complacência pulmonar, ocasionando um desequilíbrio ventilação- perfusão e diminuição do volume pulmonar, o que pode levar a situações críticas, como hipoxemia grave. Segundo alguns estudos realizados, as principais características patológicas que podem ser encontradas em pacientes 
acometidos pelo COVID-19 incluem exsudação, infiltração fibrinoso nas vias respiratórias, principalmente nas regiões distais, não causando sinais típicos de SDRA, o que pode explicar a má reposta de pacientes críticos com o COVID-19 ao recrutamento pulmonar e pressão expiratória final positiva (PEEP), em associação com a ventilação mecânica. Logo, a oxigenoterapia e a ventilação mecânica pode não resolver totalmente essa patologia relacionada a SDRA, decorrente do COVID-19, pelos seus mecanismos de funcionamento. A posição prona pode mobilizar secreç̃oes copiosas e promover a drenagem anterior em direção às vias aéreas centrais para desobstrução. Além disso, ela pode melhorar a homogeneidade pulmonar, a redistribuição pulmonar, a complacência pulmonar, a proporção de recrutamento para infiltração e o recrutamento de pulmão.

Para a SDRA grave causada por COVID-19, é recomendado a posição prona para os pacientes críticos em ventilação mecânica. Essa terapia é de intervenção eficaz no tratamento precoce de pacientes com sintomas críticos e/ou grave acometido pela doença. Assim, o uso de decúbito ventral e decúbito lateral em pacientes em estágio inicial da doença exigiu menos uso dos recursos médicos, sugerindo que os riscos de gerar novas lesões e / ou complicações são baixos. Sendo assim, pode-se considerar benéfico isso como uma intervenção precoce (Dong, et al., 2020).

Apesar do valor posionamento prono nos pacientes com SDRA moderada a grave seja convincente, ainda não se sabe ao certo a respeito dos efeitos do posicionamento prono nos pacientes adultos não intubados com respiração espontânea. Alguns estudos demonstraram a segurança e melhorias na oxigenação com a posição prona em pacientes com a síndrome. Os não intubados com COVID-19, esta técnica junto com uma estratégia combinada de cânula nasal de alto fluxo, e fluido restritivo, ou ventilação não invasiva, melhorou a oxigenação. Considerável número de pacientes apresentou melhora significativa do padrão respiratório durante o período inicial de posicionamento em pronação. É improvável que o mecanismo potencial de benefício do posicionamento prono em pacientes não intubados esteja relacionado apenas à melhora da oxigenação, sendo que em alguns estudos não se relacionam a melhora da oxigenação a sobrevida na SDRA. A aeração pulmonar homogênea com a posição prona, pode resultar em uma redução do esforço respiratório e levar a uma menor incidência de intubação. Diante disso, dado o potencial do posionamento propenso com uma intervenção de baixo custo, facilmente implementada e escalável, particularmente em países de baixa e média renda, testes rápidos, porém completos, do posionamento propenso em pacientes em risco de intubação são necessários (Damarla, M. et al., 2020).

A posição prona é um método de tratamento para o manejo de pacientes com SDRA, podendo ser usada como uma terapia coadjuvante para melhoria da ventilação e consequentemente sobrevida do paciente. A seleção correta dos pacientes e a aplicação do protocolo de tratamento adequado para o posicionamento prono são fundamentais para sua eficácia. Além da eficácia desse método, os aspectos de cuidados e os efeitos colaterais dessa posição em pacientes com SDRA também devem ser considerados. De acordo com alguns estudos, a posição prona pode diminuir a mortalidade desses pacientes quando realizada nas horas iniciais da manifestação da doença, em pacientes com saturação prejudicada e por um longo tempo. A duração mínima sugerida dessa posição é aproximadamente 12 horas por dia (Ghelichkhani P., Esmaeili M., 2020).

Com a análise de alguns estudos, percebe-se que a maioria deles demonstram que essa posição oferece benefícios a esse paciente. Pode-se incluir nas condutas terapêuticas e de suporte ventilatório aos pacientes com SDRA, acometidos pelo COVID-19. Assim, consegue-se atenuar os índices de mortalidade, dessa população que necessita de cuidados.

\section{Conclusão}

Conforme o que foi abordado durante essa revisão, conclui-se que a posição de prona proporciona diversos benefícios para os pacientes não-intubados com COVID-19, pois pode melhorar a oxigenação em curto prazo e adiar ou prevenir a necessidade de intubação e reduzir a mortalidade.

Todavia, observou-se também que esse processo carece de muita atenção dos profissionais de saúde, visto que a 
infecção pelo novo corona vírus ainda é uma patologia pouco conhecida, cheia de desafios e alterações inesperadas.

Sugere-se para trabalhos futuros, abordar a opinião da equipe de saúde, abordar a vivência e os aspectos os quais foram adotados por esses profissionais para usar a posição de prona em pacientes não intubados, abordando as dificuldades encontradas, os critérios adotados para pronar, se realmente foi benéfico ou não, entre outros. Colocando em pauta a opinião dos profissionais de saúde, seja em relatos de casos, ou até em trabalhos transversais que evidenciam essa questão e a aborde de maneira mais detalhada.

\section{Referências}

Araújo, M. S. de, Santos, M. M. P. dos, Silva, C. J. de A., Menezes, R. M. P. de, Feijão, A. R., \& Medeiros, S. M. de. (2021). Prone positioning as an emerging tool in the care provided to patients infected with COVID-19: a scoping review. Revista Latino-Americana de Enfermagem, 29. https://doi.org/10.1590/1518-8345.4732.3397.

Bastoni, D., Poggiali, E., Vercelli, A., Demichele, E., Tinelli, V., Iannicelli, T., \& Magnacavallo, A. (2020). Prone positioning in patients treated with noninvasive ventilation for COVID-19 pneumonia in an Italian emergency department. Emerg Med J, 565-566. https://pesquisa.bvsalud.org/portal/resource/pt/mdl-32748797.

Burton-Papp, H. C., Jackson, A. I. R., Beecham, R., Ferrari, M., Nasim-Mohi, M., Grocott, M. P. W., Chambers, R., \& Dushianthan, A. (2020). Conscious prone positioning during non-invasive ventilation in COVID-19 patients: experience from a single centre. F1000Research, 9, 859. https://doi.org/10.12688/f1000research.25384.1.

Chad, T., \& Sampson, C. (2020). Prone positioning in conscious patients on medical wards: A review of the evidence and its relevance to patients with COVID-19 infection. Clinical Medicine, 20(4), e97-e103. https://doi.org/10.7861/clinmed.2020-0179.

Cohen, D., Wasserstrum, Y., Segev, A., Avaky, C., Negru, L., Turpashvili, N., Anani, S., Zilber, E., Lasman, N., Athamna, A., Segal, O., Shenhav-Saltzman, G., \& Segal, G. (2020). Beneficial effect of awake prone position in hypoxaemic patients with COVID -19: case reports and literature review. Internal Medicine Journal, 50(8), 997-1000. https://doi.org/10.1111/imj.14926.

Coppo, A., Bellani, G., Winterton, D., Di Pierro, M., Soria, A., Faverio, P., Cairo, M., Mori, S., Messinesi, G., Contro, E., Bonfanti, P., Benini, A., Valsecchi, M. G., Antolini, L., \& Foti, G. (2020). Feasibility and physiological effects of prone positioning in non-intubated patients with acute respiratory failure due to COVID-19 (PRON-COVID): a prospective cohort study. The Lancet Respiratory Medicine, 8(8), 765-774. https://doi.org/10.1016/s2213-2600(20)30268-x.

Damarla, M., Zaeh, S., Niedermeyer, S., Merck, S., Niranjan-Azadi, A., Broderick, B., \& Punjabi, N. (2020). Prone Positioning of Nonintubated Patients with COVID-19. American Journal of Respiratory and Critical Care Medicine, 202(4), 604-606. https://doi.org/10.1164/rccm.202004-1331le.

Dong, W., Gong, Y., Feng, J., Bai, L., Qing, H., Zhou, P., Du, Y., Zhu, J., \& Xu, S. (2020). Early Awake Prone and Lateral Position in Non-intubated Severe and Critical Patients with COVID-19 in Wuhan: A Respective Cohort Study. https://doi.org/10.1101/2020.05.09.20091454.

Elharrar, X., Trigui, Y., Dols, A.-M., Touchon, F., Martinez, S., Prud'homme, E., \& Papazian, L. (2020). Use of Prone Positioning in Nonintubated Patients With COVID-19 and Hypoxemic Acute Respiratory Failure. JAMA, 323(22), 2336. https://doi.org/10.1001/jama.2020.8255.

Ghelichkhani, P., \& Esmaeili, M. (2020). Prone Position in Management of COVID-19 Patients; a Commentary. Archives of Academic Emergency Medicine, 8(1), e48. https://www.ncbi.nlm.nih.gov/pmc/articles/PMC7158870/.

Ibarra-Estrada, M. Á., Marín-Rosales, M., García-Salcido, R., Aguirre-Díaz, S. A., Vargas-Obieta, A., Chávez-Peña, Q., López-Pulgarín, J. A., MijangosMéndez, J. C., \& Aguirre-Avalos, G. (2020). Prone positioning in non-intubated patients with COVID-19 associated acute respiratory failure, the PRO-CARF trial: A structured summary of a study protocol for a randomised controlled trial. Trials, 21(1). https://doi.org/10.1186/s13063-020-04882-2.

Instituto de efectividad clinica y sanitaria, IECS. (2020). Posicionamento en prono en infección por COVID-19. Documento de Evaluación de Tecnologías Sanitarias - Informe de Respuesta Rápida $n^{\circ} 787$. https://www.iecs.org.ar/wp-content/uploads/IECS-IRR-787-Posicionamiento-en-prono-en-COVID-19.pdf.

Jiang, L. G., LeBaron, J., Bodnar, D., Caputo, N. D., Chang, B. P., Chiricolo, G., Flores, S., Kenny, J., Melville, L., Sayan, O. R., Sharma, M., Shemesh, A., Suh, E., \& Farmer, B. (2020). Conscious Proning: An Introduction of a Proning Protocol for Nonintubated, Awake, Hypoxic Emergency Department COVID-19 Patients. Academic Emergency Medicine, 27(7), 566-569. https://doi.org/10.1111/acem.14035.

Moghadam, V. D., Shafiee, H., Ghorbani, M., \& Heidarifar, R. (2020). Prone positioning in management of COVID-19 hospitalized patients. Brazilian Journal of Anesthesiology (English Edition), 70(2), 188-190. https://doi.org/10.1016/j.bjane.2020.05.001.

Paul, V., Patel, S., Royse, M., Odish, M., Malhotra, A., \& Koenig, S. (2020). Proning in Non-Intubated (PINI) in Times of COVID-19: Case Series and a Review. Journal of Intensive Care Medicine, 35(8), 818-824. https://doi.org/10.1177/0885066620934801.

Petrone, P., Brathwaite, C. E. M., \& Joseph, D. K. (2020). Prone ventilation as treatment of acute respiratory distress syndrome related to COVID-19. European Journal of Trauma and Emergency Surgery. https://doi.org/10.1007/s00068-020-01542-7.

Raoof, S., Nava, S., Carpati, C., \& Hill, N. S. (2019). High-Flow, Noninvasive Ventilation and Awake (Nonintubation) Proning in Patients With Coronavirus Disease 2019 With Respiratory Failure. Chest, 1992-2002. https://pesquisa.bvsalud.org/portal/resource/pt/mdl-32681847.

Sartini, C., Tresoldi, M., Scarpellini, P., Tettamanti, A., Carcò, F., Landoni, G., \& Zangrillo, A. (2020). Respiratory Parameters in Patients With COVID-19 After Using Noninvasive Ventilation in the Prone Position Outside the Intensive Care Unit. JAMA, 2338-2340. https://pesquisa.bvsalud.org/portal/resource/pt/mdl-32412606. 
Research, Society and Development, v. 10, n. 6, e38810615910, 2021

(CC BY 4.0) | ISSN 2525-3409 | DOI: http://dx.doi.org/10.33448/rsd-v10i6.15910

Souza, Marcela Tavares de, Silva, Michelly Dias da, \& Carvalho, Rachel de. (2010). Revisão integrativa: o que é e como fazer. Einstein (São Paulo), 8(1), 102-106. https://doi.org/10.1590/s1679-45082010rw1134.

Wendt, C., Mobus, K., Weiner, D., Eskin, B., \& Allegra, J. R. (2021). Prone Positioning of Patients With Coronavirus Disease 2019 Who Are Nonintubated in Hypoxic Respiratory Distress: Single-Site Retrospective Health Records Review. Journal of Emergency Nursing, 47(2), 279-287.e1. https://doi.org/10.1016/j.jen.2020.12.006.

Whittemore, P., Macfarlane, L., Herbert, A., \& Farrant, J. (2020). Use of awake proning to avoid invasive ventilation in a patient with severe COVID-19 pneumonitis. BMJ Case Reports, 13(8), e236586. https://doi.org/10.1136/bcr-2020-236586.

Winck, J. C., \& Ambrosino, N. (2020). COVID-19 pandemic and non invasive respiratory management: Every Goliath needs a David. An evidence based evaluation of problems. Pulmonology, 26(4), 213-220. https://doi.org/10.1016/j.pulmoe.2020.04.013. 